\title{
Análise de fatores de risco de queda em idosos internados em um hospital terciário no sul do Brasil
}

\author{
Alice Kalsing ${ }^{\star}$, Gabriela Guimarães Oliveira*, Ingrid Schierholt da Silva**, Jéssika Cefrin Dantas Neris ${ }^{\star *}$, \\ Mara Regina Knorst ${ }^{\star * *}$, Janete de Souza Urbanetto****
}

\section{Resumo}

Para analisar os fatores de risco de queda em pacientes geriátricos internados em um hospital terciário no sul do Brasil foi realizada uma pesquisa quantitativa, do tipo coorte. Participaram do estudo, 86 idosos hospitalizados em duas unidades de internação (geriátrica e clínica médica), de maio a outubro de 2014. Foram realizadas duas avaliações em cada paciente, no primeiro dia de internação (até 24 horas) e após 72 horas. As variáveis analisadas foram sexo, idade, força muscular (escala de Rossi e Mistrorigo), capacidade funcional (índice de Katz) e risco para quedas (Morse fall scale). A análise foi realizada pela estatística descritiva. Do total de pacientes avaliados, $45(52,3 \%)$ eram do sexo feminino e $41(47,7 \%)$ do sexo masculino. Desses, quarenta sujeitos $(46,5 \%)$ estavam na faixa etária de 60 a 79 anos e 46 (53,5\%), na faixa de 80 a 95 anos. Durante o período de avaliação, encontrou-se um declínio nos percentuais da força muscular, o índice de Katz apontou para maior dependência dos pacientes e a Morse fall scale mostrou um aumento dos idosos com elevado risco de quedas, sendo que cerca de $70 \%$ dos sujeitos possuíam elevado risco. As análises da Morse fall scale, com idade, sexo, força muscular, e o índice de Katz mostraram associações estatisticamente significantes $(p \leq 0.05)$. O sexo feminino, a idade avançada, a perda de força muscular e a dependência moderada são fatores estatisticamente significativos para risco de quedas.

Palavras-chave: Acidentes por quedas. Hospitalização. Idoso. Medição de risco.

\section{Introdução}

\section{O envelhecimento pode ser compreen-} dido como um processo natural e dinâmico de modificações progressivas morfológi-

* Fisioterapeutas. Residentes em Saúde do Idoso no Hospital São Lucas, da Pontifícia Universidade Católica do Rio Grande do Sul. Endereço para correspondência: Avenida Wenceslau Escobar, 1.086, Porto Alegre. Rio Grande do Sul. E-mail: alicekalsing@hotmail.com; oliveira_gabriela@hotmail.com

** Enfermeiras. Residentes em Saúde do Idoso no Hospital São Lucas, da Pontifícia Universidade Católica do Rio Grande do Sul. E-mail: ingridss88@gmail.com; jeg3@hotmail.com

**** Fisioterapeuta. Doutora em Gerontologia Biomédica pela Pontifícia Universidade Católica do Rio Grande do Sul. Professora da Faculdade de Enfermagem, Nutrição e Fisioterapia da Pontifícia Universidade Católica do Rio Grande do Sul. E-mail: mknorst@pucrs.br

**** Enfermeira. Doutora em Ciências da Saúde pela Pontifícia Universidade Católica do Rio Grande do Sul. Professora da Faculdade de Enfermagem, Nutrição e Fisioterapia da Pontifícia Universidade Católica do Rio Grande do Sul. E-mail: jurbanetto@pucrs.br

$\rightarrow$ http://dx.doi.org/10.5335/rbceh.v13i1.5733

Recebido em: 06/02/2016. Aceito em: 24/06/2016. 
cas, bioquímicas, psicológicas e funcionais dos indivíduos - senescência. Em condições de estresse, como uma queda, isso pode ocasionar uma condição patológica que requeira assistência - senilidade (FREITAS; PY, 2012). Diante dessas alterações, e dando ênfase à redução da capacidade funcional, idosos mostram-se mais propensos a sofrer quedas (GASPAROTTO; FALSARELLA; COIMBRA, 2014; FULLER, 2000; SILVA et al., 2013).

A capacidade funcional $(\mathrm{CF})$ pode ser definida como um parâmetro de qualidade de vida nessa população, expressada pela habilidade de manter e realizar atividades básicas de vida diária (ABVD) e atividades instrumentais de vida diária, necessárias para uma vida independente e autônoma (FREITAS; PY, 2012; GASPAROTTO; FALSARELLA; COIMBRA, 2014). Queda pode ser definida como um evento não intencional, provocado por circunstâncias multifatoriais, em que o corpo se desloca inesperadamente para o chão ou a um nível inferior à sua posição inicial (SOCIEDADE BRASILEIRA DE GERIATRIA E GERONTOLOGIA, 2008; ORGANIZAÇÃO MUNDIAL DA SAÚDE, 2007).

Segundo dados da Organização Mundial da Saúde (OMS), a frequência de quedas está estatisticamente ligada ao aumento da idade cronológica e ao nível de fragilidade, sendo que $28-35 \%$ das pessoas com 65 anos ou mais caem a cada ano, aumentando para $32-42 \%$ para aqueles com mais de 70 anos de idade (OMS, 2007).

Durante o período de internação hospitalar, as alterações fisiológicas próprias do processo de envelhecimento tornam-se mais suscetíveis aos eventos adversos da hospitalização, tais como a restrição ao leito e perda de massas óssea e muscular, restrição física, polifarmácia e procedimentos terapêuticos, alterações nutricionais, privação sensorial, comorbidades agudas ou crônicas descompensadas. Essas complicações podem levar a um declínio funcional e, consequentemente, à maior instabilidade postural e ao risco de quedas, à maior morbimortalidade $\mathrm{e}$ também a um aumento do tempo de permanência hospitalar (SALES et al., 2010).

A hospitalização aumenta o risco de quedas (SEVERO et al., 2014; DYKES et al. 2010; CORREA et al., 2012). As taxas de quedas podem variar de 1,4 a 17,9 , para cada mil pacientes-dia, dependendo do tipo de hospital, perfil e motivo da admissão dos pacientes (PRATES et al., 2014; SCHWENDIMANN et al., 2006). A literatura aponta dados relevantes: entre $15 \%$ e $50 \%$ das quedas resultam em uma grande variedade de danos, sendo que de $1 \%$ a $10 \%$ podem ser casos mais graves, com lacerações, lesões da cabeça e fraturas (CORREA et al., 2012; NASSAR; HELOU; MADI, 2013). Quedas podem resultar no aumento do tempo de permanência e consequentemente, dos custos hospitalares (SCHWENDIMANN et al., 2006; HAINES et al., 2013).

Nesse contexto, para Neto, Raso e Brito (2015), a perda de massa muscular que está associada a uma série de disfunções e doenças sistêmicas prevalentes no idoso altera a funcionalidade muscular. Dessa forma, a força muscular passa a representar um importante preditor de mobilidade funcional e risco de queda em idosos. 
A partir dos dados apresentados, considerando que idosos mostram-se mais propensos a sofrer quedas e que a hospitalização aumenta esse risco, torna-se imprescindível que os profissionais da saúde busquem ferramentas que possam auxiliar na escolha e no planejamento de intervenções preventivas, buscando excelência no cuidado. De acordo com dados da OMS e da Sociedade Brasileira de Geriatria e Gerontologia, o processo de prevenção de quedas começa com uma avaliação do risco de queda de cada paciente (SOCIEDADE BRASILEIRA DE GERIATRIA E GERONTOLOGIA, 2008; ORGANIZAÇÃO MUNDIAL DA SAÚDE, 2007).

Dessa forma, a proposta deste estudo é fazer uma análise dos fatores de risco de queda, utilizando os instrumentos índice de Katz, Morse fall scale (MFS) e um teste de força muscular (FM), escala de Rossi e Mistrorigo. Este estudo tem como objetivo avaliar o risco de queda em pacientes geriátricos internados em um hospital terciário no Sul do Brasil.

\section{Métodos}

Trata-se de uma pesquisa quantitativa, do tipo coorte, realizada em um hospital de grande porte da região metropolitana de Porto Alegre, Rio Grande do Sul, Brasil, no período de maio a outubro de 2014.

A amostra do estudo foi de idosos hospitalizados em duas unidades de internação (geriátrica e clínica médica), que tinham como critério de inclusão ter idade $\geq 60$ anos e ser avaliados por meio de um instrumento de coleta pelos pesquisadores em até 24 horas do início da internação. $O$ critério de exclusão foi não ter condições de resposta verbal em função de algum agravo à saúde.

A coleta dos dados foi realizada por uma equipe capacitada, com a utilização de um instrumento de coleta e manual orientador, com treinamento prévio. Foram realizadas duas avaliações em cada paciente incluído. Uma no primeiro dia da hospitalização, e outra, se paciente ainda hospitalizado, três dias após a primeira. Foi estabelecido esse intervalo de tempo em função da média de permanência de internação dos idosos no hospital, que é em torno de seis dias. Dessa forma, estabeleceu-se o intervalo de 72 horas entre a primeira e a segunda avaliação para abranger o máximo de idosos na segunda avaliação. As variáveis analisadas neste estudo foram sexo, idade, FM (escala de Rossi e Mistrorigo) (KENDALL et al., 2007), CF (índice de Katz) (DUARTE; ANDRADE; LEBRÃO, 2007) e risco para quedas (Morse fall scale, traduzida e adaptada transculturalmente para o português) (URBANETTO et al., 2013).

A FM foi avaliada pela aplicação da Escala de Rossi e Mistrorigo nos dois momentos de avaliação. Foram avaliados os membros superiores e inferiores direto e esquerdo. Essa escala pontua grau 5 (normal ou 100\%): movimento articular é completo e possui força suficiente para vencer a gravidade e a grande resistência aplicada; grau 4 (bom ou 75\%): o movimento é completo e com força suficiente para vencer a gravidade e alguma resistência aplicada; grau 3 (regular ou 
50\%): o movimento é completo e sua força é suficiente para vencer apenas a gravidade; grau 2 (pobre ou 25\%): o movimento é completo, mas só produz movimento se não houver ação da gravidade; grau 1 (traço ou 10\%): há evidência de pequenas contrações, mas não aciona a articulação; grau 0 (zero ou $0 \%$ ): não há evidência de contração muscular. A pontuação foi dicotomizada em força preservada (5), força muscular reduzida (4 a 2) e força muscular ausente (1 a 0 ).

$\mathrm{O}$ indice de Katz é composto por seis itens avaliativos: banho, vestir, ir ao banheiro, transferência, continência e alimentação. Neste estudo, utilizou-se a versão de 1963, que classifica os pacientes em dependência completa, dependência parcial e independência e a versão de 1989, com classificações e pontuações: muito dependente $(2,1,0$ pontos), dependência moderada ( $5,4,3$ pontos) e independente ( 6 pontos).

A Morse fall scale, na versão em língua portuguesa contém seis itens de avaliação: a) histórico de queda (sim, caiu nos últimos três meses, 25 pontos; não caiu nos últimos três meses, zero pontos); b) diagnóstico secundário, com uma ou mais patologias (sim, possui mais de um diagnóstico, 15 pontos, não possui mais de um diagnóstico, zero pontos); c) auxílio na deambulação (não utiliza, zero pontos, totalmente acamado, zero pontos, deambula auxiliado por profissional de saúde, zero pontos, usa muleta/bengala/andador, 15 pontos, segura-se no mobiliário/parede 30 pontos); d) terapia endovenosa/dispositivo endovenoso salinizado ou heparinizado (não faz uso, zero pontos, sim, faz uso, 20 pontos); e) marcha (normal zero pontos, não deambula/totalmente acamado, zero pontos, usa cadeira de rodas, zero pontos, marcha fraca, 10 pontos, marcha comprometida/cambaleante, 20 pontos); f) estado mental (orientado/capaz quanto à sua capacidade/limitação, zero pontos, superestima capacidade/esquece limitações, 15 pontos). A soma das pontuações de cada item gera um escore para a classificação do risco em risco baixo $(0-24$ pontos), risco moderado ( $25-44$ pontos), risco elevado ( $\geq 45$ pontos).

Os dados foram inseridos em um banco de dados, utilizando uma planilha eletrônica Excel. A análise foi realizada no Statistical Package for the Social Sciences (SPSS 17.0), utilizando a estatística descritiva (frequência absoluta e relativa e de variabilidade) e analítica (teste Qui-Quadrado ou Fischer) para avaliação da associação entre as variáveis. Para critérios de decisão, foi adotado o nível de significância de 5\%.

$\mathrm{O}$ projeto de pesquisa foi aprovado pelo Comitê de Ética em Pesquisa da Pontifícia Universidade Católica do Rio Grande do Sul (OF. CEP 1272/09) e os participantes assinaram termo de consentimento livre e esclarecido.

\section{Resultados}

Durante o período de estudo, 86 pacientes hospitalizados foram avaliados em até 24 horas após sua entrada na unidade de internação (primeira avaliação) e reavaliados após 72 horas (segunda avaliação). Houve uma per- 
da amostral de 14 idosos na segunda avaliação, devido à alta hospitalar, a óbito e a complicações de saúde que impossibilitaram a aplicação da segunda avaliação.

Do total de pacientes, $45(52,3 \%)$ eram do sexo feminino e $41(47,7 \%)$ do sexo masculino. Desses, 40 sujeitos $(46,5 \%)$ estavam na faixa etária de 60 a
79 anos e $46(53,5 \%)$ na faixa de 80 a 95 anos. A média da idade encontrada foi de $78,57 \pm 9,58$ anos, com mediana de 81 anos, idade mínima de 60 e máxima de 95 anos.

A análise da FM (Tabela 1) mostrou que entre o primeiro e o terceiro dia de avaliação, os percentuais da força muscular preservada reduziram e os da força muscular reduzida aumentaram.

Tabela 1 - Avaliação da força muscular

\begin{tabular}{l|r|r}
\hline \multicolumn{1}{c|}{ Variáveis } & $\begin{array}{r}\text { Primeira avaliação } \\
\mathrm{n}=86\end{array}$ & $\begin{array}{c}\text { Segunda avaliação } \\
\mathrm{n}=72\end{array}$ \\
\cline { 2 - 3 } & $\mathrm{n}(\%)$ & $\mathrm{n}(\%)$ \\
\hline Força muscular membro superior direito & $33(38,4)$ & $24(33,3)$ \\
Força preservada & $49(57,0)$ & $45(62,5)$ \\
Força muscular reduzida & $4(4,7)$ & $5(6,9)$ \\
Força muscular ausente & & \\
Força muscular membro superior esquerdo & $33(38,4)$ & $25(34,7)$ \\
Força preservada & $48(55,8)$ & $43(59,7)$ \\
Força muscular reduzida & $5(5,8)$ & $4(5,5)$ \\
Força muscular ausente & & $25(34,7)$ \\
Força muscular membro inferior direito & $34(39,5)$ & $43(59,7)$ \\
Força preservada & $47(54,7)$ & $4(5,5)$ \\
Força muscular reduzida & $5(5,8)$ & \\
Força muscular ausente & & $23(31,9)$ \\
Força muscular membro inferior esquerdo & $32(37,2)$ & $45(62,5)$ \\
Força preservada & $49(57,0)$ & $4(5,5)$ \\
Força muscular reduzida & $5(5,8)$ & \\
Força muscular ausente &
\end{tabular}

Fonte: elaboração dos autores.

A análise do índice de Katz (Tabela 2), também nesse período, mostrou um aumento dos pacientes classificados como muito dependentes e com dependência moderada e uma redução dos classificados como independentes. Ao analisar as seis atividades avaliadas, nas categorias banho, vestir e ir ao banheiro, encontrou-se um aumento na dependência completa e independência e uma redução na dependência parcial. Nas atividades de transferência e alimentação, a dependência completa e dependência parcial reduziram e a independência aumentou. Quanto à continência, a dependência completa e independência reduziram e a dependência parcial aumentou. 
Tabela 2 - Avaliação do índice de Katz e Morse fall scale

\begin{tabular}{|c|c|c|}
\hline \multirow[t]{2}{*}{ Variáveis } & $\begin{array}{l}\text { Primeira avaliação } \\
n=86\end{array}$ & $\begin{array}{c}\text { Segunda avaliação } \\
n=72\end{array}$ \\
\hline & $\mathrm{n}(\%)$ & $\mathrm{n}(\%)$ \\
\hline \multicolumn{3}{|l|}{ ÍNDICE DE KATZ- Banho } \\
\hline Dependência completa & $37(43,0)$ & $34(54,1)$ \\
\hline Dependência parcial & $19(22,1)$ & $10(13,8)$ \\
\hline Independência & $30(34,9)$ & $28(38,9)$ \\
\hline \multicolumn{3}{|l|}{ ÍNDICE DE KATZ - Vestir } \\
\hline Dependência completa & $30(34,9)$ & $28(38,9)$ \\
\hline Dependência parcial & $22(25,6)$ & $12(16,6)$ \\
\hline Independência & $34(39,5)$ & $32(44,4)$ \\
\hline \multicolumn{3}{|l|}{ ÍNDICE DE KATZ - Ir ao banheiro } \\
\hline Dependência completa & $22(25,6)$ & $20(27,8)$ \\
\hline Dependência parcial & $27(31,4)$ & $18(25,0)$ \\
\hline Independência & $37(43,0)$ & $34(54,1)$ \\
\hline \multicolumn{3}{|l|}{ ÍNDICE DE KATZ - Transferência } \\
\hline Dependência completa & $16(18,6)$ & $12(16,6)$ \\
\hline Dependência parcial & $26(30,2)$ & $16(22,2)$ \\
\hline Independência & $44(51,2)$ & $44(61,1)$ \\
\hline \multicolumn{3}{|l|}{ ÍNDICE DE KATZ - Continência } \\
\hline Dependência completa & $20(23,3)$ & $15(20,8)$ \\
\hline Dependência parcial & $32(37,2)$ & $31(43,0)$ \\
\hline Independência & $34(39,5)$ & $26(36,1)$ \\
\hline \multicolumn{3}{|l|}{ ÍNDICE DE KATZ - Alimentação } \\
\hline Dependência completa & $17(19,8)$ & $14(19,4)$ \\
\hline Dependência parcial & $15(17,4)$ & $10(13,8)$ \\
\hline Independência & $54(62,8)$ & $48(66,6)$ \\
\hline \multicolumn{3}{|l|}{ ÍNDICE DE KATZ Total } \\
\hline Muito dependente & $20(23,3)$ & $17(23,6)$ \\
\hline Dependência moderada & $22(25,6)$ & $20(27,7)$ \\
\hline Independente & $44(51,2)$ & $35(48,6)$ \\
\hline \multicolumn{3}{|l|}{ Morse Fall Scale Total } \\
\hline Elevado risco & $62(72,1)$ & $54(75)$ \\
\hline Moderado risco & $15(17,4)$ & $12(16,6)$ \\
\hline Baixo risco & $9(10,5)$ & $6(8,3)$ \\
\hline
\end{tabular}

Fonte: elaboração dos autores.

AMFS mostrou que cerca de $70 \%$ dos pacientes apresentaram elevado risco de queda. Entre o primeiro e terceiro dia de avaliação, houve um aumento do per- centual de pacientes com elevado risco bem como uma redução do percentual de pacientes com moderado e baixo risco de queda (Tabela 2). 
Na Tabela 3, na associação entre MFS e sexo, verificaram-se porcentagens maiores na classificação de elevado risco para ambos os sexos ( $p=0,005)$. Quando associada a MFS com a idade, observou-se que, quanto maior a idade, maior o risco de queda, de 60 a 79 anos, $57,5 \%$, e de 80 a 95 anos, $87,0 \%$ ( $p=0,007)$. No cruzamento da MFS com a FM, encontrou- -se associação significativa para todos os membros avaliados, mostrando que, quanto menor a força apresentada, maior o risco de queda. A avaliação da MFS e o índice Katz mostram que pacientes muito dependentes $(81,0 \%)$ e com dependência moderada $(95,8 \%)$ estão associados ao maior risco de cair do que pacientes independentes $(56,1 \%)(\mathrm{p}=0,007)$.

Tabela 3 - Associação da Morse fall scale com sexo, idade, força muscular e índice de Katz

\begin{tabular}{|c|c|c|c|c|c|c|}
\hline & & \multirow{2}{*}{\multicolumn{3}{|c|}{ MFS }} & \multirow[b]{3}{*}{${ }^{*} \mathrm{P}$} & \multirow[b]{3}{*}{ Total } \\
\hline & & & & & & \\
\hline & & $\begin{array}{l}\text { Baixo } \\
\text { risco }\end{array}$ & $\begin{array}{l}\text { Moderado } \\
\text { risco }\end{array}$ & $\begin{array}{c}\text { Elevado } \\
\text { risco }\end{array}$ & & \\
\hline \multirow[t]{2}{*}{ Sexo } & Feminino & $0(0,0)$ & $8(17,8)$ & $37(82,2)$ & \multirow{3}{*}{,005 } & $45(100,0)$ \\
\hline & Masculino & $8(19,5)$ & $7(17,1)$ & $26(63,4)$ & & $41(100,0)$ \\
\hline \multirow[t]{2}{*}{ Idade } & 60 a 79 anos & $6(15,0)$ & $11(27,5)$ & $23(57,5)$ & & $40(100,0)$ \\
\hline & 80 a 95 anos & $2(4,3)$ & $4(8,7)$ & $40(87,0)$ & \multirow[t]{2}{*}{,007 } & $46(100,0)$ \\
\hline \multirow{3}{*}{$\begin{array}{l}\text { FM } \\
\text { MSD }\end{array}$} & Preservada & $6(18,2)$ & $11(33,3)$ & $16(48,5)$ & & $33(100,0)$ \\
\hline & Reduzida & $2(4,2)$ & $4(8,3)$ & $42(87,5)$ & \multirow[t]{2}{*}{,001 } & $48(100,0)$ \\
\hline & Ausente & $0(0,0)$ & $0(0,0)$ & $5(100,0)$ & & $5(100,0)$ \\
\hline \multirow{3}{*}{$\begin{array}{l}\text { FM } \\
\text { MSE }\end{array}$} & Preservada & $6(18,2)$ & $11(33,3)$ & $16(50,0)$ & \multirow{3}{*}{, 000} & $33(100,0)$ \\
\hline & Reduzida & $2(4,2)$ & $4(8,3)$ & $42(87,2)$ & & $48(100,0)$ \\
\hline & Ausente & $0(0,0)$ & $0(0,0)$ & $5(100,0)$ & & $5(100,0)$ \\
\hline \multirow{3}{*}{$\begin{array}{l}\text { FM } \\
\text { MID }\end{array}$} & Preservada & $6(18,2)$ & $11(33,3)$ & $17(48,5)$ & \multirow{3}{*}{,001 } & $34(100,0)$ \\
\hline & Reduzida & $2(4,2)$ & $4(8,3)$ & $41(87,5)$ & & $47(100,0)$ \\
\hline & Ausente & $0(0,0)$ & $0(0,0)$ & $5(100,0)$ & & $5(100,0)$ \\
\hline \multirow{3}{*}{$\begin{array}{l}\text { FM } \\
\text { MIE }\end{array}$} & Preservada & $6(18,2)$ & $11(33,3)$ & $17(48,5)$ & \multirow{3}{*}{, 003} & $34(100,0)$ \\
\hline & Reduzida & $2(4,2)$ & $4(8,3)$ & $41(87,5)$ & & $47(100,0)$ \\
\hline & Ausente & $0(0,0)$ & $0(0,0)$ & $5(100,0)$ & & $5(100,0)$ \\
\hline \multirow{3}{*}{$\begin{array}{l}\text { Índice } \\
\text { de Katz }\end{array}$} & Independente & $7(17,1)$ & $11(26,8)$ & $23(56,1)$ & \multirow{3}{*}{,007 } & $41(100,0)$ \\
\hline & Dependência Moderada & $0(0,0)$ & $1(4,2)$ & $23(95,8)$ & & $24(100,0)$ \\
\hline & Muito Dependente & $1(4,8)$ & $3(14,3)$ & $17(81,0)$ & & $21(100,0)$ \\
\hline
\end{tabular}

Fonte: elaboração dos autores.

Nota: FM - força muscular; MSD/MSE - membro superior direito/membro superior esquerdo; MID/MIE - membro inferior direito/membro inferior esquerdo. * Teste de Fisher.

\section{Discussão}

Neste estudo foi evidenciado que 0 maior percentual de pacientes avaliados eram do sexo feminino $(52,3 \%)$ e a faixa etária predominante ficou no grupo de 80 a 95 anos (53,5\%). Segundo os índices do Instituto Brasileiro de Geografia e Estatística, a expectativa de vida do bra- 
sileiro ao nascer passou de 74,1, em 2011, para 74,6 anos, em 2012, com mulheres vivendo em torno de 78,3 anos e homens 71 anos (INSTITUTO BRASILEIRO DE GEOGRAFIA E ESTATÍSTICA, 2010). Tal situação corrobora os dados encontrados, revelando maiores percentuais de mulheres hospitalizadas e com idades mais avançadas.

O envelhecimento populacional, com todos os fatores que lhe estão associados, faz com que esses indivíduos careçam de maior atenção em cuidados e, muitas vezes, procurem atendimento de profissionais de saúde, necessitando de hospitalização. Idosos representam uma importante demanda nas internações hospitalares, apresentando tempo de internação maior, quando comparados a outras faixas etárias (SALES et al., 2010). Estudo realizado por Schwendimann et al. (2006) chamou a atenção para um aumento de pacientes do sexo feminino hospitalizados, com idades mais avançadas, no período de 1999 a 2003.

Estudos mostram efeitos devastadores do repouso no leito sobre a FM de idosos (CREDITOR, 1993; SUESADA; MARTINS; CARVALHO, 2007). A publicação que avaliou os perigos da hospitalização para idosos relatou que a massa muscular reduz com o envelhecimento, esse fato somado ao aumento da inatividade física, reflete-se na perda progressiva de FM e, consequentemente, na capacidade de reserva biológica. A contração muscular com força e frequência mínima é necessária para manter a FM, e na privação de contrações voluntárias, a FM reduz em 5\% ao dia. Homens jovens restritos ao leito perdem de $1,0 \%$ a $1,5 \%$ de FM ao dia, podendo chegar a $10 \%$ por semana (CREDITOR, 1993). Os dados encontrados em nosso estudo reforçam os achados apresentados, pois, identificou-se uma redução importante dos percentuais de FM para os membros avaliados.

Suesada, Martins e Carvalho (2007) avaliaram os efeitos da internação de curto prazo, e identificaram que antes e após um dia de hospitalização ocorreu uma redução da FM dos membros superiores e, consequentemente, da $\mathrm{CF}$, mesmo em pacientes que não estavam restritos ao leito, independente da idade ou do estado funcional inicial. Nota-se que os dois artigos usaram indivíduos jovens, podendo-se prever que os efeitos seriam ainda mais significativos em indivíduos idosos (CREDITOR, 1993; SUESADA; MARTINS; CARVALHO, 2007).

Com relação ao índice de Katz, a amostra apontou para um aumento significativo na dependência dos pacientes avaliados. No estudo realizado por Zelada, Salinas e Baztán (2009), ao avaliarem a incidência de deteriorização funcional de idosos internados em unidades geriátricas, comparada a uma unidade de terapia convencional, identificaram que pacientes idosos apresentaram declínio funcional após hospitalização. Outro estudo que avaliou a incidência e os fatores associados ao comprometimento funcional de idosos hospitalizados, comparando unidades geriátricas e de clínica médica, identificou declínio funcional durante a hospitalização, observada em $48,1 \%$ dos pacientes no serviço de geria- 
tria e $60,2 \%$ dos pacientes em serviço de clínica médica (ASTIZ et al., 2008).

O declínio funcional apresentado durante o período de hospitalização não é consequência exclusiva de comorbidades, mas, também, dos processos que envolvem a internação. Ressalta-se que de $30 \%$ a $60 \%$ dos idosos hospitalizados sofrem perda da CF e a prevenção é um importante alvo para todos os trabalhadores de saúde, assim a identificação de pacientes de alto risco é o primeiro passo para a prevenção (HOOGERDUIJN et al., 2012).

$\mathrm{O}$ índice de Katz foi desenvolvido a partir de estudos que mostraram uma hierarquização de respostas neurológicas e locomotoras das atividades conforme sua complexidade de realização, semelhante à sequência observada no processo de desenvolvimento da criança (DUARTE; ANDRADE; LEBRÃO, 2007). Nossos achados corroboram esses dados, pois banhar-se, vestir-se e ir ao banheiro foram as tarefas em que demonstrou-se maior dependência, enquanto que os atos de continência, transferência e alimentação, exigiram menor dependência.

A classificação conforme a MFS apontou que os pacientes estavam em maior percentual classificados com elevado risco de quedas. Esses resultados corroboram os achados encontrados por $\mathrm{Al}$ Tehewy et al. (2015) que avaliou pacientes hospitalizados, por meio da MFS e constatou que a escala foi capaz de prever os pacientes em risco de cair, identificando corretamente $82,6 \%$ das quedas.

Foi possível verificar que houve um aumento nos escores de elevado risco de queda, avaliados pela MFS, da primeira para a última avaliação. Esse dado reforça a necessidade de avaliação do paciente no momento da admissão e realização de avaliações subsequentes. O Ministério da Saúde (MS) orienta que a avaliação seja feita diariamente até a alta do paciente, reforçando a reavaliação sempre que houver a identificação de outros fatores de risco como transferências de setor, mudança do quadro clínico e quando houver um episódio de queda. Assim, estratégias devem ser implantadas com o objetivo de prevenção (BRASIL, 2013).

Buscando constatar uma relação entre a variável sexo e o risco de queda, encontrou-se para ambos os sexos um valor significativo, mas com maior percentual para o feminino. Prates et al. (2014), ao identificar a incidência e as características das quedas em adultos hospitalizados, não encontraram diferença entre o sexo masculino e o feminino, entre os pacientes que sofreram quedas.

No presente estudo, encontrou-se diferença significativa entre os sexos, o que corrobora os dados apresentados pela OMS, em que mulheres são mais propensas a cair e a sofrer lesões mais fatais do que os homens. Fatores biológicos podem explicar esse aumento do risco, pois as mulheres têm uma importante redução da massa óssea após a menopausa e têm, também, menor quantidade de massa magra e força muscular do que os homens, o que, em parte, explica essa maior fragilidade e predisposição a sofrer quedas (SILVA et al., 2013; ORGANIZAÇÃO MUNDIAL DA SAÚDE, 2007).

$\mathrm{Na}$ associação da idade com a MFS, observou-se que, quanto maior a idade, 
maior o risco de quedas, encontrou-se $57,5 \%$ de risco de quedas entre os pacientes de 60 a 79 anos e $87 \%$ entre os de 80 a 95 anos. Prates et al. (2014) relataram que a maioria dos pacientes estudados $(90,8 \%)$ apresentavam risco para quedas e a idade $\geq 65$ anos foi o principal fator (81,5\%). O avanço da idade, associado a um declínio funcional, já se apresenta como um importante fator de risco para quedas, e esse fator evidencia-se durante um processo de hospitalização, quando há um aumento das limitações físicas e psicológicas e, consequentemente, um risco elevado para quedas.

Encontrou-se percentuais de FM reduzida em todos os membros, estatisticamente associada ao aumento do risco de quedas. Diccini, Pinho e Silva (2008) relataram que entre pacientes neurológicos, essa variável está fortemente relacionada ao risco de queda e à queda. $\mathrm{O}$ estudo apontou que dos pacientes que caíram antes de internar, $52,3 \%$ relataram como causa a diminuição da FM.

Segundo dados da OMS, pessoas com fraqueza muscular são quase cinco vezes mais propensas a cair. Além disso, indivíduos com menor FM de extremidades, medida pela extensão do joelho e flexão dorsal do tornozelo, têm 1,8 vezes maior risco de queda e três vezes mais risco de quedas recorrentes. Podemos, então, dizer que a redução da FM é um fator de risco para queda (ORGANIZAÇÃO MUNDIAL DA SAÚDE, 2007).

Encontrou-se, neste estudo, uma associação significativa da MFS com o índice de Katz. Estudo realizado por Almeida (2012) avaliou os fatores de risco para queda, utilizando a escala de Morse e a escala de Downton e encontrou forte associação estatística do risco de queda com a necessidade de ajuda na realização das ABVD ( $p=0,000)$. Esse foi o único estudo encontrado em que se associaram as variáveis escala de Morse e índice de Katz. Outros estudos podem ser encontrados com o objetivo de identificar pacientes com risco de quedas a partir da CF. Esses estudos encontraram resultados semelhantes, mesmo utilizando diferentes ferramentas de avaliação (SILVA, 2013; QUEIROZ; LIRAC; SASAK, 2009).

A OMS não traz a CF como um fator de risco para queda, a informação divulgada é de que queda pode causar medo de cair, reduzir a independência e a qualidade de vida, gerando a síndrome pós-queda, que está associada a uma perda de confiança e à consequente imobilidade (ORGANIZAÇÃO MUNDIAL DA SAÚDE, 2014). Já o Ministério da Saúde apresenta a dificuldade na realização das ABVD como um fator de risco para quedas (BRASIL, 2013). De acordo com dados da Sociedade Brasileira de Geriatria e Gerontologia, o risco de quedas aumenta progressivamente conforme $o$ grau de dependência do indivíduo (SOCIEDADE BRASILEIRA DE GERIATRIA E GERONTOLOGIA, 2008).

Outro fato observado na população estudada foi que o risco de queda é maior em pacientes com dependência moderada do que em pacientes muito dependentes. Esse dado corrobora os apontados pelo Ministério da Saúde, que referem pacientes com alto risco de 
queda, os independentes, que têm pelo menos um fator de risco, o dependente, com ou sem fator de risco, o que anda com auxílio (de pessoa ou de dispositivo) e o paciente acomodado em maca (por exemplo, aguardando exames ou transferência), com ou sem a presença de fatores de risco. Já o paciente com baixo risco de queda é o acamado, restrito ao leito, completamente dependente, com ou sem fatores de risco, e o indivíduo totalmente independente e sem nenhum fator de risco (BRASIL, 2013).

$\mathrm{O}$ uso de protocolos e indicadores de avaliação são ferramentas importantes no processo de melhoria da qualidade e da segurança na assistência ao paciente. Prevenir quedas sempre será a opção mais eficaz e barata. Estudos mostram que as intervenções multifacetadas tendem a ser mais efetivas na busca da prevenção, envolvendo a educação contínua de pacientes, familiares e profissionais de saúde (HAINES et al., 2013; FULLER, 2000).

Muitos fatores relacionados ao risco de quedas são passíveis de intervenções, como a implantação de unidades geriátricas, equipes multidisciplinares, avaliações recorrentes, comunicação efetiva entre profissionais, uso racional de medicamentos, entre outros, reduzindo a incidência de declínio funcional e impactando de forma favorável na qualidade de vida desses pacientes.

\section{Conclusão}

O processo de envelhecimento culmina em alterações estruturais e funcionais que se acumulam de forma progressiva com o passar dos anos. Tais alterações comprometem o desempenho de habilidades motoras, dificultam a adaptação do indivíduo ao ambiente e o predispõe à queda. A diminuição da FM, presente no processo de envelhecimento leva o indivíduo a uma dependência funcional que também predispõe o indivíduo a este evento.

A partir do presente estudo, foi possível concluir que o sexo feminino, a idade avançada, a perda de FM e a dependência moderada são realmente fatores que predispõem para o risco de queda.

A contribuição deste estudo para a área da saúde consiste na efetiva identificação de grupos com maiores fatores de risco para queda, permitindo redirecionar aos profissionais da saúde o planejamento de estratégias voltadas para a segurança desses pacientes. Dessa forma, retardando alterações advindas do processo de envelhecimento e déficit funcionais pela prevenção.

O estudo apresenta limitações que devem ser discutidas, quanto ao número de pacientes avaliados e ao número de quedas ocorridas na amostra durante a pesquisa, o que dificultou algumas análises estatísticas.

\section{Analysis the risk factors to falls in elderly patients in tertiary care hospital in southern Brazil}

\section{Abstract}

To analyze the risk factors to falls in elderly patients admitted to a tertiary care hospital in Southern Brazil. Quantitative research, the cohort. Study includes 86 elderly hospitalized in two inpatient units (Geriatric 
and Internal Medicine) from May to October 2014. Two evaluations were performed on each patient on the first day of hospitalization (within 24 hours) and after 72 hours. The variables were gender, age, muscle strength (Rossi Scale and Mistrorigo), functional capacity (Katz Index), and risk of falls (Morse Fall Scale). The analysis was performed using descriptive statistics. From the patients evaluated, 45 (52.3\%) were female and $41(47.7 \%)$ were male. From these, 40 subjects $(46.5 \%)$ were aged $60-79$ years and $46(53.5 \%)$ in the range 80-95 years. During the evaluation, there was a decline in the percentage of muscle strength, the Katz Index pointed to a greater reliance on patients and Morse Fall Scale showed an increase of high risk of falls in the elderly, of which about $70 \%$ of the subjects had high risk. The analysis of Morse Fall Scale with age, gender, muscle strength and Katz Index showed associations statistically significant $(p \leq 0.05)$. The female gender, advanced age, loss of muscle strength and moderate dependence are statistically significant factors to the risk of falls.

Keywords: Accidental falls. Hospitalization. Elderly. Risk assessment.

\section{Referências}

AL TEHEWY, M. M.; AMIN, G. E.; NASSAR, N. W. A study of rate and predictors of fall among elderly patients in a university hospital. Journal of Patient Safety, v. 11, n. 4, p. 210-214, Dec. 2015.

ALMEIDA, C. I. S. Risco de quedas na UCCI do distrito de Viseu: estudo comparativo escala de Morse e escala de Downton. 2012. Dissertação (Mestrado em Educação para a Saúde) - Instituto Politécnico de Viseu, Escola Superior de Saúde de Viseu, Viseu, 2012.

ASTIZ, M. T. V. et al. Deterioro functional durante la hospilatización en ancianos. Beneficios del ingreso en el servicio de geriatria. Revista Española de Geriatría y Gerontología, v. 43, n. 3, p. 133-138, maio 2008.
BRASIL. Ministério da Saúde. Anexo 1: Protocolo de Prevenção de Quedas. 2013. Programa Nacional de Segurança do Paciente. Disponível em: <http://www.hospitalsantalucinda.com.br/downloads/prot_quedas.pdf $>$. Acesso em: 16 nov. 2014.

CORREA, A. D. et al. Implantação de um protocolo para gerenciamento de quedas em hospital: resultado de quatro anos de seguimento. Revista da Escola de Enfermagem da USP, São Paulo, v. 14, n. 46, p. 67-74, fev. 2012.

CREDITOR, M. C. Hazards of hospitalization of elderly. Annals of Internal Medicine, v. 118, n. 3, p. 219-23, fev. 1993.

DICCINI, S.; PINHO, P.; SILVA, F. O. Avaliação de risco e incidência de queda em pacientes neurocirúrgicos. Revista Latino- Americana de Enfermagem, Ribeirão Preto, v. 16, n. 4, p. 1-6, jul./ago. 2008.

DUARTE, Y. A. O.; ANDRADE, C. L.; LEBRÃO, M. L. Index de Katz na avaliação da funcionalidade dos idosos. Revista da Escola de Enfermagem da USP, São Paulo, v. 41, n. 2, p. 317-325, jun. 2007.

DYKES, P. C. et al. Fall prevention in acute care hospitals. JAMA, v. 304, n. 17, p. 1.9121.918, nov. 2010.

FREITAS, E. V.; PY, L. Tratado de geriatria e gerontologia. Rio de Janeiro: Guanabara Koogan; 2012.

FULLER, G. F. Falls in the elderly. American Family Physician, v. 61, n. 7, p. 2.159-2.168, abr. 2000.

GASPAROTTO, L. P. R.; FALSARELLA, G. R.; COIMBRA, A. M. V. As quedas no cenário da velhice: conceitos básicos e atualidades da pesquisa em saúde. Revista Brasileira de Geriatria e Gerontologia, Rio de Janeiro, v. 17, n. 1, p. 201-209, jan./mar. 2014.

HAINES, T. P. et al. Cost effectiveness of patient education for the prevention of falls in hospital: economic evaluation from a randomized controlled trial. BMC Medicine, v. 11, n. 135, p. 1-4, May 2013. 
HOOGERDUIJN, J. G. et al. The prediction of functional decline in older hospitalised patients. Age and Ageing, v. 41, n. 3, p. 381387, Feb. 2012.

INSTITUTO BRASILEIRO DE GEOGRAFIA E ESTATÍSTICA. Grandes regiões e unidades da federação: esperança de vida ao nascer segundo projeção populacional 1980, 1991-2030 - ambos os sexos. 2010. Disponível em: <http://www.ibge.gov.br/home/presidencia/noticias/imprensa/ppts/0000000243.pdf $>$. Acesso em: 16 nov. 2014.

KENDALL, P. et al. Músculos: provas e funções. 5. ed. São Paulo: Barueri Manole; 2007.

NASSAR, N.; HELOU, N.; MADI, C. Predicting falls using two instruments (the Hendrich Fall Risck Model and the Morse Fall Scale) in an acute care setting in Lebanon. Journal of Clinical Nursing, v. 14, n. 23, p. 1.620-1.629, jun. 2013.

NETO, J. P.; RASO, W.; BRITO, C. A. F. Mobilidade funcional em função da força muscular em mulheres idosas fisicamente ativas. Revista Brasileira de Medicina do Esporte, v. 21, n. 5. p. 369-371, set./out. 2015 ORGANIZAÇÃO MUNDIAL DA SAÚDE. A Global report on falls prevention: epidemiology of falls. 2007. Disponível em: <http://www. who.int/ageing/publications/Falls_prevention7March.pdf>. Acesso em: 16 nov. 2014.

PRATES, C. G. et al. Quedas em adultos hospitalizados: incidência e características desses eventos. Ciência, Cuidado e Saúde, Maringá, v. 14, n. 13, p. 74-81, jan./mar. 2014.

QUEIROZ, L.; LIRAC, S.; SASAK, A. Identificação do risco de quedas pela avaliação da mobilidade funcional em idosos hospitalizados. Revista Baiana de Saúde pública, Salvador, v. 33, n. 4, p. 534-543, out./dez. 2009.

SALES, M. V. C. et al. Efeitos adversos da internação hospitalar para o idoso. Geriatria \& Gerontologia, Rio de Janeiro, v. 4, n. 4, p. 238-246, out./dez. 2010.
SCHWENDIMANN, R. et al. Falls and consequent injuries in hospitalized patients: effects of an interdisciplinary falls prevention program. BMC Health Services Research, v. 6 , n. 69 , p. $1-7$, jun. 2006.

SEVERO, I. M. et al. Fatores de risco para quedas em pacientes adultos hospitalizados: revisão integrativa. Revista da Escola de Enfermagem da USP, São Paulo, v. 48, n. 3, p. 540-554, maio/jun. 2014.

SILVA, J. M. N. et al. Correlação entre o risco de queda e autonomia funcional em idosos institucionalizados. Revista Brasileira de Geriatria e Gerontologia, Rio de Janeiro, v. 16, n. 2, p. 337-346, abr./jun. 2013.

SOCIEDADE BRASILEIRA DE GERIATRIA E GERONTOLOGIA. Quedas em idosos: prevenção. 2008. Disponível em: <http:// www.projetodiretrizes.org.br/projeto_diretrizes/082.pdf>. Acesso em: 16 nov. 2014.

SUESADA, M. M.; MARTINS, M. A.; CARVALHO, C. R. Effect of short-term hospitalization on functional capacity in patients not restricted to bed. American Journal of Physical Medicine \& Rehabilitation, v. 86, n. 6, p. 455-62, jun. 2007.

URBANETTO, J. S. et al. Morse fall scale: tradução e adaptação transcultural para a língua portuguesa. Revista da Escola de Enfermagem da USP, São Paulo, v. 47, n. 3, p. 569-575, jun. 2013.

ZELADA, M. A.; SALINAS, R.; BAZTÁN, J. J. Reduction of functional deterioration during hospitalization in an acute geriatric unit. Archives of Gerontology and Geriatrics, v. 48, n. 1, p. 35-39, Jan./Feb. 2009. 